\title{
Fair Resource Allocation with Interference Mitigation and Resource Reuse for LTE/LTE-A Femtocell Networks
}

\author{
Ying Loong Lee, Student Member, IEEE, Jonathan Loo, Member, IEEE, Teong Chee Chuah, \\ and Ayman A. El-Saleh, Member, IEEE
}

\begin{abstract}
Joint consideration of interference, resource utilization, fairness and complexity issues is generally lacking in existing resource allocation schemes for Long Term Evolution (LTE)/LTEAdvanced femtocell networks. To tackle this, we employ a hybrid spectrum allocation approach whereby the spectrum is split between the macrocell and its nearby interfering femtocells based on their resource demands, while the distant femtocells share the entire spectrum. A multi-objective problem is formulated for resource allocation between femtocells and is decomposed using a lexicographic optimization approach into two subproblems. A reasonably low-complexity greedy algorithm is proposed to solve these subproblems sequentially. Simulation results show that the proposed scheme achieves substantial throughput and packet loss improvements in low-density femtocell deployment scenarios while performing satisfactorily in high-density femtocell deployment scenarios with substantial complexity and overhead reduction. The proposed scheme also performs nearly as well as the optimal solution obtained by exhaustive search.
\end{abstract}

Index Terms-LTE/LTE-Advanced, femtocell, hybrid spectrum allocation, resource allocation, interference, resource utilization, fairness.

\section{INTRODUCTION}

$\mathbf{F}$ EMTOCELLS have emerged as a promising solution to minimize indoor coverage holes and to cope with the increasing indoor traffic demands in Long Term Evolution (LTE)/LTE-Advanced (LTE-A) networks [1]. In LTE/LTE-A ${ }^{1}$ networks, femtocells are deployed with low-power base stations known as home evolved NodeBs (HeNBs). These HeNBs are installed in a plug-and-play manner and are connected to the operator's core network via indoor broadband wirelines such as the digital subscriber line (DSL) [2]. As femtocell deployment is simple and of low-cost, it is more favorable than redimensioning macrocells for providing better connectivity to the indoor user equipment (UE).

It has been shown in [3] that substantial throughput gains can be obtained by deploying femtocells within macrocells

Y. L. Lee, T. C. Chuah and A. A. El-Saleh are with the Faculty of Engineering, Multimedia University, 63100 Cyberjaya, Selangor, Malaysia e-mail: (lee.ying.loong12@student.mmu.edu.my, \{tcchuah, ayman.elsaleh\}@mmu.edu.my).

J. Loo is with the School of Science and Technology, Middlesex University, London NW4 4BT, United Kingdom e-mail: (J.Loo@mdx.ac.uk).

This work is supported in part by the Ministry of Higher Education Malaysia under the Fundamental Research Grant Scheme MMUE/140082.

${ }^{1}$ The 'LTE/LTE-A' terminology is used because both LTE and LTE-A share the same fundamental architecture and our work applies to both standards. Hence, the use of this terminology arises. Hereafter, this terminology is used whenever the common aspects, features, architectures, etc. of both LTE and LTE-A are discussed. with co-channel frequency reuse. However, optimal throughput performance cannot be achieved due to the coexistence of macrocells and femtocells which results in cross-tier interference [4]. Furthermore, femtocells could be randomly deployed by the end users and some of them may overlap with one another, thereby resulting in co-tier interference [4]. As a result, the throughput gains achieved from femtocell deployment may be significantly limited. Several enhanced intercell interference coordination (eICIC) schemes have been introduced in LTEA to mitigate cross-tier interference [5], [6]. However, the eICIC schemes do not address the co-tier interference, fairness and resource utilization issues. To address these issues, the resource allocation scheme should be further improved by jointly optimizing throughput performance (by mitigating the interference problems), fairness and resource utilization efficiency.

In the literature, resource allocation for femtocell networks has been intensively studied under the shared spectrum [7][13] and split spectrum [14]-[20] approaches. The shared spectrum approach allows the macrocell and femtocells to share the channel bandwidth. However, this approach cannot fully mitigate cross-tier interference, thus limiting the achievable capacity. Also, the shared spectrum approach may require a coordination mechanism between the central macrocell base station (also known as the evolved NodeB (eNB)) and HeNBs, which may introduce scalability and security issues [20], [21]. In the split spectrum approach, the channel bandwidth is divided among the macrocell and femtocells, which entirely avoids cross-tier interference and results in a straightforward resource allocation process. However, the split spectrum approach provides less available bandwidth to the macrocell and femtocells respectively, resulting in lower achievable capacity. In this work, we employ a hybrid spectrum allocation approach whereby the eNB and its nearby interfering HeNBs operate under the split spectrum approach whereas the distant HeNBs operate under the shared spectrum approach.

In LTE/LTE-A systems, real-time traffic flows such as video and voice flows fall under the guaranteed bit rate (GBR) class, which imposes certain resource demands to guarantee the quality of service (QoS), e.g., minimum target bit rates. On the other hand, nonreal-time traffic flows such as best-effort delivery are grouped under the non-GBR class, which does not impose stringent QoS requirements and the achievable data rate depends on the availability of resources. When each HeNB receives the amount of resources it requires for its GBR and 
non-GBR flows, the resource allocation is said to be globally fair [1]. However, most of the related work in [7]-[17], [19], [20] only considered the GBR class. To ensure a high level of global fairness, it is crucial to ensure that each HeNB has its minimum resource demand satisfied for the GBR flows, and be provided with a fair amount of resources for the non-GBR flows. In this work, we aim to achieve globally fair resource allocation, catering for both GBR and non-GBR flows.

In practice, the channel bandwidth given may not be sufficient to meet the resource demands of femtocell networks if the resources are assigned to each femtocell in a dedicated manner without resource reuse. Thus, resource reuse is essential whereby the resources allocated by some femtocells can be reused in other femtocells without interference. However, it is important to study the interference relationships between femtocells before planning for an efficient resource reuse. In this work, we consider the use of an HeNB management system (HMS) [22]-[24], which connects to a group of neighboring HeNBs and acts as a central controller to collect interference information from all the HeNBs and to perform resource allocation.

In this paper, we propose a multi-objective resource allocation scheme under a hybrid spectrum allocation approach with reasonable complexity for LTE/LTE-A femtocell networks to jointly optimize network performance. The proposed scheme can be implemented in both open-access and closed-access femtocells. The main contributions of this paper can be summarized as follows:

1) A hybrid spectrum allocation approach is used for spectrum allocation between the macrocell and femtocells, whereby the spectrum is split between the eNB and its nearby interfering HeNBs based on their resource demands; while the distant HeNBs are allocated the entire spectrum.

2) A multi-objective optimization problem is formulated for resource allocation between femtocells to jointly minimize co-tier interference and maximize resource utilization efficiency, subject to the resource demand and global fairness constraints. The problem is further decomposed using a lexicographic optimization approach into an interference minimization subproblem and a resource utilization maximization subproblem. The solution obtained from solving these two subproblems sequentially is proven to be Pareto optimal for the original multi-objective problem.

3) A two-phase greedy algorithm is devised to solve the two subproblems sequentially. The complexity and amount of signaling overhead of the proposed scheme are shown to be reasonably low compared to those of the existing schemes.

4) The proposed resource allocation scheme is evaluated and compared with the existing schemes in terms of throughput, packet loss rate (PLR), global fairness and resource utilization efficiency in low-density and highdensity femtocell deployment scenarios with different number of UEs in both macrocell and femtocells. Also, the solution obtained by the proposed greedy algorithm is also compared with the optimal solution obtained by exhaustive search.

Hereafter, our work is studied based on the LTE context and system settings due to the following reasons: 1) The proposed scheme is not intended to be dependent on the features introduced in LTE-A such as eICIC and should generally be applicable to both LTE and LTE-A systems; 2) For the proposed scheme to be applicable to both LTE and LTE-A systems, it is appropriate to study the proposed scheme mainly based on the LTE context and settings because LTE is the base architecture of LTE-A; 3) The new features introduced in LTE-A such as eICIC can be complementary to our proposed resource allocation scheme for interference mitigation, which is not necessary to be studied together with our proposed scheme; 4) Without loss of generality and for the clarity of performance outcomes, it is appropriate to evaluate and analyze our proposed scheme without involving the new features introduced in LTE-A such as eICIC. Although our work is based on the LTE context and system settings, the proposed scheme is generally applicable to any orthogonal frequency division multiple access (OFDMA)-based small-cell network.

The remainder of this paper is organized as follows: Section II reviews the related work. Section III describes the LTE/LTEA femtocell system model. Spectrum allocation between the macrocell and femtocells is presented in Section IV. Section $\mathrm{V}$ presents the problem formulation for resource allocation between femtocells and the solution algorithm proposed for the formulated problem. Section VI analyzes and compares the complexity and signaling overhead of the proposed scheme with existing schemes. Performance evaluation of the proposed scheme is presented in Section VII. Finally, Section VIII provides the concluding remarks.

\section{RELATED WORK}

A comprehensive survey of various resource allocation techniques for heterogeneous LTE/LTE-A networks can be found in [1]. In this section, the recent related work is reviewed next.

Several recent studies [7]-[13] have investigated the shared spectrum approach for femtocell networks. In [7], the entire channel bandwidth is assigned to macrocell UEs (MUEs), while a portion of it is assigned to femtocell UEs (FUEs), the size of which is adjusted based on a spectrum splitting ratio. In addition, a max-min fair scheduler is used. However, a bruteforce approach may be required to find the optimal ratio, which leads to high complexity, and cross-tier interference could still remain.

In [8], the channel bandwidth is partitioned into two sets of resources for serving indoor and outdoor MUEs respectively. The size of the two sets depends on the instantaneous indoor traffic load generated by indoor MUEs. FUEs are allocated resources iteratively based on a stochastic rule until their resource demands are fulfilled. However, this only reduces cross-tier interference and co-tier interference still remains.

Several authors [9], [10] have studied the use of a central controller for shared resource allocation in femtocell networks. In [9], the so-called centralized dynamic frequency planning 
(C-DFP) is proposed to allocate resources to femtocells to meet their minimum resource demands based on the interference from their neighboring femtocells and the macrocell. In [10], the central controller models the interference among the macrocell and femtocells as a link-conflict graph, and solves it using a graph coloring technique that minimizes the number of resources allocated. However, in both [9] and [10], maximization of resource utilization efficiency is not considered and the computational complexity could be very high if the number of femtocells is large.

In [11]-[13], cognitive radio technologies are leveraged for shared spectrum allocation between macrocells and femtocells. In [11], HeNBs identify and utilize unoccupied spectrum via spectrum sensing and the scheduling information from the eNB. In [12], HeNBs perform spectrum sensing to identify free resources and share the resources using a non-cooperative game theoretic strategy. In [13], the macrocell and femtocell share the spectrum using a cooperative coalitional game strategy and a decentralized coalition formation algorithm. Although cognitive radio technologies are promising, their implementation remains challenging because additional hardware is required in the eNB and HeNBs [20].

Several recent resource allocation schemes [14]-[20] are developed under the split spectrum approach. In [14], the channel bandwidth is split between the macrocell and femtocell based on their respective numbers of users. To avoid co-tier interference, the so-called distributed random access (DRA) scheme is proposed to allocate resources among the femtocells based on their number of interfering neighbors by using a randomized hashing function and a collision avoidance mechanism. The complexity of the DRA scheme is relatively low due to its decentralized implementation. However, global fairness is not considered in the study.

In [15] and [16], power control techniques are used with the split spectrum approach to reduce co-tier interference. In [15], all the femtocells share the channel bandwidth using proportional fair scheduling and perform power control using an enhanced iterative water-filling method. The power level is estimated based on the interference and traffic information exchanged between the femtocells. In [16], resources are allocated in a way such that the resource demands of HeNBs are fulfilled with minimum transmission power. However, co-tier interference may not be fully eliminated using the techniques in [15] and [16], and global fairness and maximization of resource utilization efficiency are not considered in [15].

Several split spectrum schemes [17], [18] centralize the resource allocation among femtocells in one single network controller. In [17], a centralized proportional fair scheduler is used to allocate resources among all the FUEs. On the other hand, the HMS is used in [18] as a central controller for each group of neighboring femtocells and allocates resources to the FUEs based on their resource demands using a greedy approach. In addition, the resources allocated to an FUE can be reused by another FUE if they are not interfering with each other, resulting in high resource utilization efficiency. However, both schemes assume that the network controller performs resource allocation for each FUE instead of each HeNB. The computational complexity can be prohibitively high when the number of FUEs is too large.

In [19], a chordal graph approach is used to estimate the amount of resources to be granted to each femtocell and resource allocation among femtocells is performed based on greedy graph coloring. Fairness is further guaranteed by using a logarithmic utility function to maximize data rate. However, global fairness is still lacking. Also, different traffic classes with different QoS requirements are not considered in the study.

In [20], clustering is performed to form groups with each consisting of femtocells that interfere with each other. Each group selects an HeNB as the cluster head to perform resource allocation among the femtocells. Resource allocation is performed by taking into account the QoS requirements imposed by each FUE associated to the femtocells in the group. Although the clustering approach significantly reduces complexity compared to centralized approaches, the computational burden lies on the HeNB that serves as the cluster head. As HeNBs are in general equipped with low processing power, the clustering approach still incurs high complexity.

In general, the techniques proposed in [7]-[20] do not jointly consider interference, global fairness, resource utilization and complexity issues. Furthermore, most of these techniques are not designed to meet the QoS requirements of both GBR and non-GBR flows as defined in the LTE/LTE-A standard. Also, both the shared and split spectrum approaches can be capacity-limited due to higher interference and less bandwidth allocated, respectively. To strike a tradeoff between these two approaches, a hybrid spectrum allocation approach which combines both approaches has been proposed in [25]. In this approach, the HeNBs which are near the eNB operate under the split spectrum approach whereas those which are far from the eNB operate under the shared spectrum approach. On the other hand, the eNB operates under the split spectrum approach if there exist nearby interfering HeNBs; otherwise, it operates under the shared spectrum approach. The hybrid spectrum allocation approach is promising but other issues such as global fairness and resource utilization are not addressed. Motivated by these observations, the current work develops a resource allocation scheme under the hybrid spectrum allocation approach to jointly address the interference, resource utilization and fairness problems with reasonable complexity.

\section{SySTEM MODEL}

We consider a system model shown in Fig. $1^{2}$ wherein each group of neighboring HeNBs forms a femtocell group and connects to an HMS [22]-[24]. In reality, the HeNBs in a femtocell group can be those that are deployed within the same indoor building. Let $\mathcal{H}$ denote the set of all HeNBs, $\mathcal{L}$ denote the set of HMSs, $\mathcal{H}_{l}$ denotes the set of HeNBs associated with HMS $l$ and $\mathcal{U}_{h}$ denotes the set of FUEs being served by HeNB $h$. It is noteworthy that $\mathcal{H}=\bigcup_{l \in \mathcal{L}} \mathcal{H}_{l}$. In LTE/LTE-A systems, the channel bandwidth is divided into

\footnotetext{
${ }^{2}$ The HeNBs in each femtocell group actually connect to the HMS via a security gateway [22]-[24]. As the security gateway does not play a significant role in our system model, we omitted its illustration in Fig. 1. For a detailed illustration of the LTE/LTE-A femtocell network architecture, the reader may refer to [24].
} 


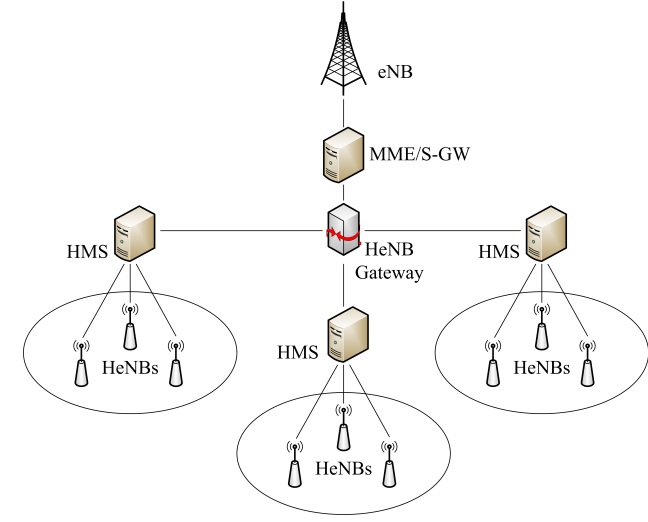

Fig. 1. LTE/LTE-A femtocell network model.

a number of subchannels based on the OFDMA technology. Each subchannel has a bandwidth of $180 \mathrm{kHz}$ and is further divided into a number of time blocks known as physical resource blocks (PRBs) [26] each lasting a duration of 0.5 ms. In LTE/LTE-A systems, resources are allocated to UEs every one transmission time interval (TTI). Thus, at least two PRBs are allocated to a UE during each TTI. Our proposed scheme works by allocating subchannels in such a way that the HeNBs exploit all the PRBs from the subchannels allocated. As such, let $\mathcal{K}$ denotes the set of subchannels available to the femtocell network and $\mathcal{K}_{l}$ denotes the set of subchannels allocated to the femtocell group associated with HMS $l$. It is noteworthy that $\mathcal{K}=\bigcup_{l \in \mathcal{L}} \mathcal{K}_{l}$. Without loss of generality, we make the following assumptions: 1) The bandwidth of each subchannel is less than the coherence bandwidth, i.e., each subchannel experiences flat fading; 2) The duration of each PRB is shorter than the coherence time, i.e., each PRB experiences slow fading; and 3) The femtocell network is perfectly synchronized.

In LTE/LTE-A standards, two types of data flows are defined: GBR and non-GBR. GBR flows have strict QoS requirements whereas non-GBR flows do not need strict QoS guarantees. Each HeNB may carry a number of GBR and non-GBR flows. As such, we denote $\mathcal{C}_{\mathrm{GBR}, h}$ and $\mathcal{C}_{\mathrm{nG}, h}$ as the respective sets of GBR flows and non-GBR flows carried by HeNB $h$. Each GBR flow requires a specific minimum bit rate to attain minimum QoS satisfaction. The minimum resource demand for achieving the required minimum bit rate of a GBR flow can be estimated by:

$$
D_{c}=\left\lceil\frac{R_{\mathrm{req}, c}}{f_{\mathrm{PRB}} E}\right\rceil \quad \forall c \in \bigcup_{h \in \mathcal{H}} \mathcal{C}_{\mathrm{GBR}, h}
$$

where $D_{c}$ is the minimum number of subchannels required by flow $c, R_{\text {req }, c}$ is the minimum bit rate required by flow $c, f_{\mathrm{PRB}}$ $=180 \mathrm{kHz}$ is the bandwidth of a PRB (or a subchannel), and $E$ is the achievable spectral efficiency in bits/s/Hz. The value of $E$ can be obtained using the adaptive modulation and coding (AMC) module in the medium access control (MAC) layer of the LTE/LTE-A system [27]. On the other hand, as non-GBR flows do not incur any specific minimum resource demands, it is up to the resource allocation scheme to decide the number of resources to be allocated. In this study, the minimum resource demand imposed by a non-GBR flow is set to one. The overall minimum resource demand of an HeNB can be expressed as follows:

$$
D_{h}=\sum_{c \in \mathcal{C}_{\mathrm{GBR}, h} \cup \mathcal{C}_{\mathrm{nG}, h}} D_{c}
$$

where $D_{h}$ is the minimum number of subchannels required by HeNB $h$. This resource demand information will be requested by the associated HMS for resource allocation among the femtocells.

It is noteworthy that interference between femtocell groups is negligible due to the low transmission power of HeNBs and the high penetration loss of building walls. Thus, only the interference between HeNBs within the same building, i.e., within the same group, is of interest. In a femtocell group associated with HMS $l$, the interference relationships between the HeNBs can be mathematically stated using an interference matrix, denoted as $\mathbf{A}=\left[a_{i j}\right]_{\left|\mathcal{H}_{l}\right| \times\left|\mathcal{H}_{l}\right|}$ where:

$$
a_{i j}= \begin{cases}1 & \text { if HeNB } j \text { interferes with HeNB } i \\ 0 & \text { otherwise }\end{cases}
$$

To construct the interference matrix for a femtocell group, we follow the approach in [28] whereby each FUE periodically measures the received signal strength (RSS) of the reference signals transmitted by all the HeNBs. The measurement data is then reported back to the serving HeNB to identify interfering HeNBs. If the RSS received by any FUE from an HeNB is higher than that of its serving HeNB for a certain margin, an interference link is said to exist between these two HeNBs, i.e.,

$$
a_{i j}= \begin{cases}1 & \text { if } P_{u i}(\mathrm{~dB})<P_{u j}(\mathrm{~dB})+T h(\mathrm{~dB}) \\ 0 & \text { otherwise }\end{cases}
$$

where $P_{u i}$ and $P_{u j}$ are the RSSs received by FUE $u \in \mathcal{U}_{i}$ from HeNB $i$ and HeNB $j$, respectively, and $T h$ is a protection margin that takes into account the aggregated interference from the neighboring femtocells and fading effects. After identifying the interfering HeNBs, each HeNB reports this to the HMS to construct the interference matrix.

\section{Spectrum Allocation between the Macrocell AND FEMTOCELLS}

In order to mitigate cross-tier interference and strike a tradeoff between the split and shared spectrum approaches, we adopt a hybrid approach which combines both the shared and split spectrum approaches to perform spectrum allocation between the macrocell and femtocells in an LTE/LTE-A network. Under this approach, the femtocell groups which are far from and near the eNB operate under a shared spectrum approach and a split spectrum approach, respectively. Fig. 2 illustrates the hybrid spectrum allocation model, where the distant femtocell group utilize the entire spectrum whereas the same spectrum is split between the eNB and its nearby interfering femtocell group. If there is no interfering femtocell group in the vicinity of the eNB, the eNB may utilize the entire spectrum. In fact, the hybrid approach applies to multiple femtocell groups which are near or far from the eNB. 


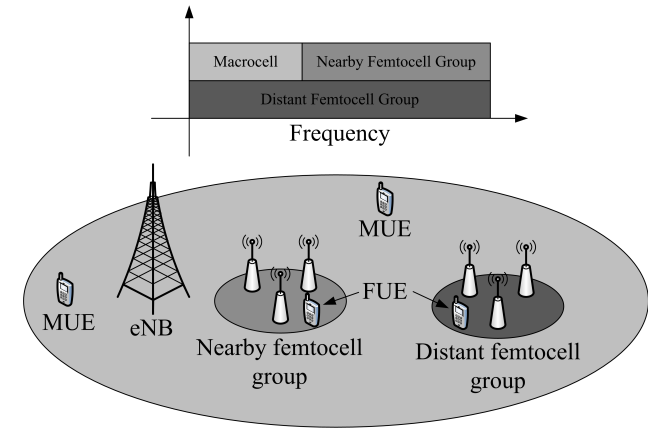

Fig. 2. Hybrid spectrum allocation model.

Unlike the hybrid spectrum allocation in [25] which splits the spectrum between the eNB and its nearby HeNBs into two fixed-size portions, our hybrid spectrum allocation adaptively splits the spectrum between the eNB and its nearby interfering femtocell groups based on their resource demands as given by

$$
\begin{gathered}
N_{\mathrm{m}}=\left\lceil\frac{D_{\mathrm{m}}}{\sum_{l \in \mathcal{L}_{\mathrm{i}}} D_{l}} \times|\mathcal{K}|\right\rceil \\
N_{\mathrm{f}}=|\mathcal{K}|-N_{\mathrm{m}}
\end{gathered}
$$

where $N_{\mathrm{m}}$ and $N_{\mathrm{f}}$ are the numbers of resources divided to the eNB and the nearby interfering femtocell groups respectively, $\mathcal{L}_{\mathrm{i}}$ is the set of HMSs connected to the interfering femtocell groups, and $D_{l}=\sum_{l h \in \mathcal{H}_{l}} D_{h}$ is the total resource demand of the femtocell group connected to HMS $l$. Note that $|\mathcal{X}|$ is the cardinality of set $\mathcal{X}$. After splitting the spectrum, the eNB receives $N_{\mathrm{m}}$ resources and all the interfering femtocell groups receive $N_{\mathrm{f}}$ resources, i.e., $\left|\mathcal{K}_{l}\right|=N_{\mathrm{f}}$ for all $l \in \mathcal{L}_{\mathrm{i}}$. Other non-interfering distant femtocell groups can utilize the entire spectrum, i.e., $\mathcal{K}_{l}=\mathcal{K}$ for all $l \in \mathcal{L} \backslash \mathcal{L}_{\mathrm{i}}$. Without loss of generality, we consider an LTE network with a femtocell group being near the eNB and a femtocell group being far from the eNB, i.e., $|\mathcal{L}|=2$ and $\left|\mathcal{L}_{\mathrm{i}}\right|=1$, in our simulation study in Section VII.

In practice, this spectrum allocation process can be performed at the eNB. The resource demand information of the interfering femtocell groups can be obtained by the eNB from the associated HMSs through the HeNB gateway [22], [24] which connects to the serving gateway [22], [24] of the eNB. We assume that the interfering femtocell groups can be identified by the eNB through their HMSs. This can be done using existing techniques such as those in [25] and [29]. When majority of the femtocells in a femtocell group are interfering with the eNB, this femtocell group is classified as a nearby interfering group to the eNB. For implementation, this hybrid spectrum allocation process should be executed every period of $T_{\text {alloc. }}$.

\section{Resource Allocation between Femtocells}

In this section, we formulate a multi-objective optimization problem for resource allocation between HeNBs in each femtocell group to jointly minimize co-tier interference and maximize resource utilization efficiency while guaranteeing a high level of global fairness. This problem is further decomposed into two subproblems which are then solved sequentially using a two-phase greedy algorithm.

\section{A. Problem Formulation}

Our objective is to minimize co-tier interference between HeNBs in each femtocell group, to attain a high level of global fairness and to maximize resource utilization efficiency. Thus, we can mathematically formulate the resource allocation problem between femtocells in a femtocell group associated with HMS $l$ as a multi-objective optimization problem, as follows:

Problem 1:

$$
\begin{array}{ll}
\min & f_{1, l}(\boldsymbol{\omega})=\frac{\sum_{i \in \mathcal{H}_{l}} \sum_{j \in \mathcal{H}_{l} \backslash\{i\}} a_{i j} \sum_{k \in \mathcal{K}_{l}} \omega_{i k} \omega_{j k}}{\left|\mathcal{K}_{l}\right|\left|\mathcal{H}_{l}\right|\left(\left|\mathcal{H}_{l}\right|-1\right)} \\
\max & f_{2, l}(\boldsymbol{\omega})=\frac{\sum_{h \in \mathcal{H}_{l}} \sum_{k \in \mathcal{K}_{l}} \omega_{h k}}{\left|\mathcal{H}_{l}\right|\left|\mathcal{K}_{l}\right|}
\end{array}
$$

subject to

$$
\begin{gathered}
\sum_{k \in \mathcal{K}_{l}} \omega_{h k}=D_{h}+N_{h} \quad \forall h \in \mathcal{H}_{l} \\
D_{c}=1 \quad \forall c \in \bigcup_{h \in \mathcal{H}_{l}} \mathcal{C}_{\mathrm{nG}, h} \\
N_{h}=S \geq 0 \quad \forall h \in \mathcal{H}_{l}
\end{gathered}
$$

where $\boldsymbol{\omega}=\left[\omega_{h k}\right]_{\left|\mathcal{H}_{l}\right| \times\left|\mathcal{K}_{l}\right|}$ with $\omega_{h k}$ being the binary assignment indicator of subchannel $k$ to $\operatorname{HeNB} h$, i.e., $\omega_{h k}=1$ if subchannel $k$ is allocated to HeNB $h$; otherwise $\omega_{h k}=0$, and $N_{h}$ is the number of additional subchannels allocated to HeNB $h$. In (7), $f_{1, l}$ is to minimize co-tier interference whereas $f_{2, l}$ is to maximize resource utilization efficiency. The denominators of both objective functions normalize the objective function values to within $[0,1]$. Constraint (7a) ensures that each HeNB receives the number of subchannels it requires, plus the number of additional subchannels. Constraint (7b) guarantees that each HeNB receives one subchannel for each of its nonGBR flows. In constraint (7c), $S$ is a positive real integer that enforces all HeNBs to receive an equal number of additional subchannels for achieving a certain level of global fairness. By maximizing resource utilization efficiency; which is equivalent to maximizing $N_{h}$ for all $h \in \mathcal{H}_{l}$, more subchannels are available for both GBR and non-GBR flows.

In multi-objective optimization, a unique global solution may not always exist due to the presence of multiple conflicting objective functions. This can be observed in Problem 1 where the minimization of co-tier interference (i.e., $f_{1, l}$ ) will reduce resource utilization efficiency (i.e., $f_{2, l}$ ). For such a problem, we can find a tradeoff solution between these conflicting objective functions in which all are optimized to a degree where no objective functions can be made better off without making the other objective functions worse off. These tradeoff solutions are said to be Pareto optimal [30]. With regard to Problem 1, Pareto optimality can be defined as follows:

Definition 1. Let $\Omega$ be the set of feasible solutions for Problem 1. A solution, $\boldsymbol{\omega}^{*} \in \Omega$ is Pareto optimal for Problem 1 if there 
does not exist another solution, $\boldsymbol{\omega} \in \boldsymbol{\Omega}$ such that $f_{1, l}(\boldsymbol{\omega}) \leq$ $f_{1, l}\left(\boldsymbol{\omega}^{*}\right)$ and $f_{2, l}(\boldsymbol{\omega})>f_{2, l}\left(\boldsymbol{\omega}^{*}\right)$, or $f_{1, l}(\boldsymbol{\omega})<f_{1, l}\left(\boldsymbol{\omega}^{*}\right)$ and $f_{2, l}(\boldsymbol{\omega}) \geq f_{2, l}\left(\boldsymbol{\omega}^{*}\right)$.

A commonly used technique to solve a multi-objective optimization problem for Pareto optimality is the weighted sum approach, which combines all the weighted objective functions to form a single-objective optimization problem. However, it is difficult to assign the appropriate weight for each objective as the solution is weight dependent [31]. On the other hand, the lexicographic optimization approach [32] is an attractive multi-objective optimization technique whereby all the objective functions are arranged and optimized, with one at a time subjecting to all constraints, in a lexicographic order, i.e., from the most important objective function to the least important one. In addition, the less important objective function will be optimized in the way such that the value of the more important objective function optimized in the previous iteration is preserved. To apply the lexicographic approach, the objective functions must be of different levels of importance. We can see that Problem 1 is one such problem, with $f_{1, l}$ being more important than $f_{2, l}$ due to the following reasons:

1) If $f_{2, l}$ is maximized first, the minimization of $f_{1, l}$ is not possible because $\omega_{h k}=0$ needs to be set for some $h$ and $k$ in order to minimize $f_{1, l}$, which reduces the value of $f_{2, l}$.

2) If $f_{1, l}$ is minimized first, the value of $f_{1, l}$ can still be preserved during the maximization of $f_{2, l}$ because $\omega_{h k}=1$ can be set for some $h$ and $k$ if $a_{h k}=0$.

If a unique solution is obtained for Problem 1 before or after all the objective functions are optimized, the solution is said to be lexicographically optimal [32]. With regard to Problem 1, lexicographic optimality can be defined as follows:

Definition 2. Let $\Omega$ be the set of feasible solutions for Problem 1 which can be expressed as a lexicographic problem where $f_{1, l}$ is more important than $f_{2, l}$. A solution, $\boldsymbol{\omega}^{*} \in \boldsymbol{\Omega}$ is lexicographically optimal for Problem 1 if there does not exist another solution, $\boldsymbol{\omega} \in \boldsymbol{\Omega}$ such that $f_{1, l}(\boldsymbol{\omega})<f_{1, l}\left(\boldsymbol{\omega}^{*}\right)$, or $f_{1, l}(\boldsymbol{\omega})=f_{1, l}\left(\boldsymbol{\omega}^{*}\right)$ and $f_{2, l}(\boldsymbol{\omega})>f_{2, l}\left(\boldsymbol{\omega}^{*}\right)$.

As Problem 1 contains two objective functions, it can be decomposed into two subproblems which are to be solved sequentially. Since $f_{1, l}$ is more important than $f_{2, l}$, the first subproblem can be expressed as the minimization of $f_{1, l}$ whereas the second subproblem can be expressed as the maximization of $f_{2, l}$. Unlike the conventional lexicographic approach, we assume that $S=0$, i.e., $N_{h}=0$ for all $h \in \mathcal{H}_{l}$ in the first subproblem to reduce its set of feasible solutions. This reduction is made because:

1) From constraints (7c), it is known that $N_{h} \geq 0$ for all $h \in \mathcal{H}_{l}$. As such, a solution to Problem 1 must at least fulfil the resource demand, $D_{h}$. This implies that $f_{2, l} \geq$ $\frac{\sum_{h \in \mathcal{H}_{l}} D_{h}}{\left|\mathcal{H}_{l}\right| \mathcal{K}_{l} \mid}$ and the maximization of $f_{2, l}$ is equivalent to maximizing $N_{h}$. Therefore, the solution must at least guarantee $f_{2, l}=\frac{\sum_{h \in \mathcal{H}_{l}} D_{h}}{\left|\mathcal{H}_{l}\right|\left|\mathcal{K}_{l}\right|}$ in a way such that $f_{1, l}$ is minimum. This suggests that such a solution must be obtained during the minimization of $f_{1, l}$. On the other hand, the maximization of $N_{h}$ can be done during the maximization of $f_{2, l}$ since both are equivalent.

2) Since any solution from the reduced feasible set for the first subproblem will give $f_{2, l}=\frac{\sum_{h \in \mathcal{H}_{l}} D_{h}}{\left|\mathcal{H}_{l}\right|\left|\mathcal{K}_{l}\right|}$, the maximization of $f_{2, l}$ in the second subproblem will only find and allocate unutilized subchannels if they do not increase the value of $f_{1, l}$. This helps reduce the search effort for a feasible solution to both subproblems.

After reducing the set of the feasible solutions for the first subproblem, constraint (7c) can be excluded and the first subproblem can be expressed as follows:

Problem 2:

$$
\min f_{1, l}(\boldsymbol{\omega})=\frac{\sum_{i \in \mathcal{H}_{l}} \sum_{j \in \mathcal{H}_{l} \backslash\{i\}} a_{i j} \sum_{k \in \mathcal{K}_{l}} \omega_{i k} \omega_{j k}}{\left|\mathcal{K}_{l}\right|\left|\mathcal{H}_{l}\right|\left(\left|\mathcal{H}_{l}\right|-1\right)}
$$

subject to constraint (7b) and

$$
\sum_{k \in \mathcal{K}_{l}} \omega_{h k}=D_{h} \quad \forall h \in \mathcal{H}_{l}
$$

where constraint (8a) is derived from constraint (7a) with $N_{h}=0$ and the new set of constraints only ensures that the minimum resource demand of each HeNB is fulfilled.

After solving Problem 2, the value of $f_{1, l}$ corresponds to the solution obtained and serves a new constraint in the second subproblem. Let $f_{1, l}^{*}$ denote the value of $f_{1, l}$ obtained from the solution found for Problem 2, the second subproblem can be expressed as follows:

Problem 3:

$$
\max \quad f_{2, l}(\boldsymbol{\omega})=\frac{\sum_{h \in \mathcal{H}_{l}} \sum_{k \in \mathcal{K}_{l}} \omega_{h k}}{\left|\mathcal{H}_{l}\right|\left|\mathcal{K}_{l}\right|}
$$

subject to constraint (7a), (7c) and

$$
f_{1, l}=f_{1, l}^{*}
$$

Constraint (9a) ensures that the solution to Problem 3 will not make the value of $f_{1, l}$ obtained from solving Problem 2 worse off. It is noteworthy that the set of feasible solutions for Problem 3 is also the one for Problem 1.

The optimal solution obtained from solving Problems 2 and 3 sequentially is in fact a Pareto optimal solution for Problem 1. This can be explained in the following propositions and the corresponding proofs:

Proposition 1. Let $\Omega$ be the set of feasible solutions for Problem 1. If $\boldsymbol{\omega}^{*} \in \boldsymbol{\Omega}$ is an optimal solution for Problem 3, it is lexicographically optimal for Problem 1.

Proof. Suppose $\boldsymbol{\omega}^{*}$ is not lexicographically optimal. Thus, there exist another solution, $\boldsymbol{\omega} \in \boldsymbol{\Omega}$ such that $f_{1, l}(\boldsymbol{\omega})<$ $f_{1, l}\left(\boldsymbol{\omega}^{*}\right)$, or $f_{1, l}(\boldsymbol{\omega})=f_{1, l}\left(\boldsymbol{\omega}^{*}\right)$ and $f_{2, l}(\boldsymbol{\omega})>f_{2, l}(\boldsymbol{\omega} *)$. By definition of Problem 2 and constraint (9a), we cannot have $f_{1, l}(\boldsymbol{\omega})<f_{1, l}\left(\boldsymbol{\omega}^{*}\right)$. Hence, it is only possible that $f_{1, l}(\boldsymbol{\omega})=f_{1, l}\left(\boldsymbol{\omega}^{*}\right)$. Therefore, $f_{2, l}(\boldsymbol{\omega})>f_{2, l}\left(\boldsymbol{\omega}^{*}\right)$ must hold if $\boldsymbol{\omega}^{*}$ is not lexicographically optimal. By definition of Problem 3, we cannot have $f_{2, l}(\boldsymbol{\omega})>f_{2, l}\left(\boldsymbol{\omega}^{*}\right)$. Thus, it is only possible that $f_{2, l}(\boldsymbol{\omega})=f_{2, l}\left(\boldsymbol{\omega}^{*}\right)$, which contradicts the assumption of $f_{2, l}(\boldsymbol{\omega})>f_{2, l}\left(\boldsymbol{\omega}^{*}\right)$. 
Proposition 2. Let $\boldsymbol{\Omega}$ be the set of feasible solutions for Problem 1. If $\boldsymbol{\omega}^{*} \in \mathbf{\Omega}$ is a lexicographically optimal solution for Problem 1, it is also Pareto optimal.

Proof. Similar to that for Proposition 1.

It is noteworthy that there can be more than one Pareto optimal solution for Problem 1. Thus, there is no guarantee that the Pareto optimal solution obtained from solving Problem 2 and Problem 3 sequentially is the best. In multi-objective optimization, any Pareto optimal solution is acceptable for the given multi-objective optimization problem [33]. In the context of this work, the best Pareto optimal solution is the one that gives the best network performance. One approach is to find a set of Pareto optimal solutions using techniques such as those in [33] and [34] for the given problem and then to evaluate each of the solutions to identify the one that gives the best network performance. However, this is not feasible for real-time applications such as resource allocation in femtocell networks due to the high computational complexity required.

\section{B. Proposed Resource Allocation Scheme}

Both Problems 2 and 3 can be solved using binary integer programming techniques. However, these techniques would make the problems NP-complete and incur high computational complexity. Thus, we propose a greedy two-phase resource allocation algorithm to efficiently find suboptimal solutions for Problems 2 and 3 with low complexity. As depicted in Algorithm 1, Phase 1 (Algorithm 2) and Phase 2 (Algorithm 3) correspond to solving Problem 2 and Problem 3 respectively.

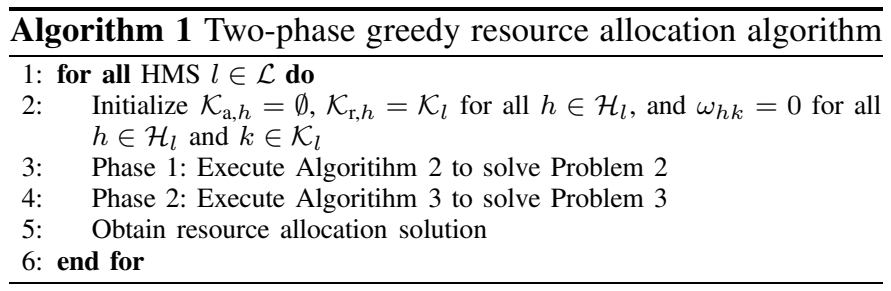

In Phase 1, subchannels are allocated to meet constraint (8a) with minimum co-tier interference. Firstly, orthogonal subchannel allocation (steps 1-5 in Algorithm 2) is performed in such a way that the number of subchannels allocated to the HeNBs is in proportion to their minimum resource demand, i.e.,

$$
P_{h}=\left\lfloor\frac{D_{h}}{\sum_{p \in \mathcal{H}_{l}} D_{p}} \times\left|\mathcal{K}_{l}\right|\right\rfloor
$$

where $P_{h}$ is the number of subchannels orthogonally allocated to HeNB $h$, and $\sum_{h \in \mathcal{H}_{l}} P_{h} \leq\left|\mathcal{K}_{l}\right|$. In this way, each HeNB $h$ receives a subset of subchannels, $\mathcal{K}_{\mathrm{a}, h}$ where $\left|\mathcal{K}_{\mathrm{a}, h}\right|=P_{h}$. This orthogonal subchannel allocation is achieved by dividing the set of subchannels, $\mathcal{K}_{l}$ into $\left|\mathcal{H}_{l}\right|$ subsets, where the first subset $\mathcal{K}_{\mathrm{a}, 1}=\left\{1,2, \ldots, P_{1}\right\}$ is allocated to HeNB 1 , the second subset $\mathcal{K}_{\mathrm{a}, 2}=\left\{P_{1}+1, P_{1}+2, \ldots, P_{1}+P_{2}\right\}$ is allocated to HeNB 2, the third subset $\mathcal{K}_{\mathrm{a}, 3}=\left\{P_{1}+P_{2}+1, P_{1}+P_{2}+2, \ldots, P_{1}+P_{2}+P_{3}\right\}$ is allocated to HeNB 3 , and so on.

The subchannels received from the orthogonal subchannel allocation may not suffice to meet constraint (8a). In the subsequent steps (steps 6-30 in Algorithm 2), a greedy algorithm

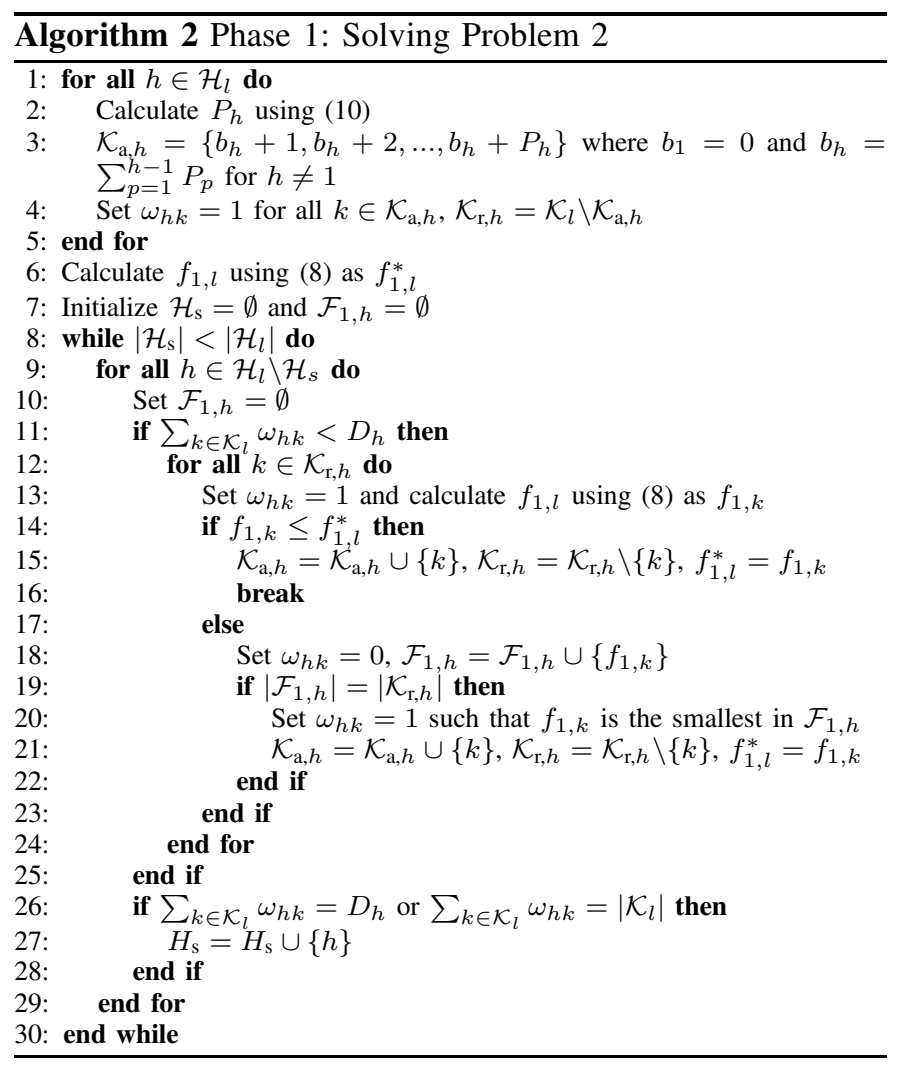

is used to allocate subchannels iteratively until the minimum resource demand of each HeNB is fulfilled. In this greedy algorithm, each HeNB is allocated one subchannel in each iteration. Let $\mathcal{K}_{\mathrm{r}, h}=\mathcal{K}_{l} \backslash \mathcal{K}_{\mathrm{a}, h}$ denote the subset of subchannels which is not allocated to HeNB $h$. A subchannel from set $\mathcal{K}_{\mathrm{r}, h}$ is allocated to HeNB $h$ if it does not inflict higher co-tier interference, i.e., $f_{1, l}$ is higher than that without the subchannel allocated. Otherwise, $f_{1, l}$ will be computed for all the subchannels in set $\mathcal{K}_{\mathrm{r}, h}$, and the subchannel which gives the lowest $f_{1, l}$ will be allocated. The allocated subchannel will be included in set $\mathcal{K}_{\mathrm{a}, h}$. This process is repeated until constraint (8a) is satisfied. It is worth noting that steps 2628 in Algorithm 2 examine whether constraint (8a) is met or all the available subchannels are allocated for each HeNB. If any of these two conditions is true, the HeNB is considered satisfied.

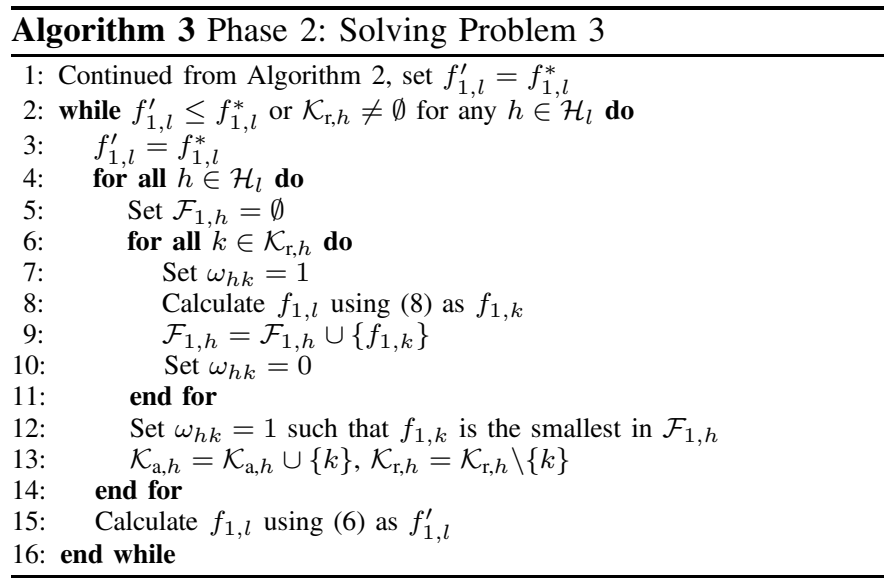


From Phase 1, the subchannel allocation solution obtained is used to calculate $f_{1, l}$ as $f_{1, l}^{*}$, which will be used to solve Problem 3. In Phase 2, we employ a similar greedy algorithm as in Phase 1 to maximize $f_{2, l}$ and meet constraints (7c) and (9a). In this greedy algorithm (see Algorithm 3), a subchannel is allocated to HeNB $h$ from set $\mathcal{K}_{\mathrm{r}, h}$ when it gives the lowest $f_{1, l}$ among other subchannels. The allocated subchannel will then be included in set $\mathcal{K}_{\mathrm{a}, h}$. Subchannel allocation is performed iteratively where each HeNB is allocated one subchannel every iteration. This is to ensure that constraint (7c) is met. After one iteration, the algorithm examines whether the new value of $f_{1, l}$, i.e., $f_{1, l}^{\prime}$ has exceeded $f_{1, l}^{*}$, and whether set $\mathcal{K}_{\mathrm{r}, h}$ is non-empty for any HeNB $h$. If both of these conditions are true, subchannel allocation will be repeated until one of them is invalid. Both conditions ensure that constraints (7c) and (9a) are met. In this way, the resource utilization efficiency in (9) is maximized.

\section{Implementation Framework}

The proposed two-phase resource allocation scheme is implemented at each HMS, and is executed periodically with an interval, $T_{\mathrm{RA}}$. At the end of each $T_{\mathrm{RA}}$, the HMS will instruct all the HeNBs to refrain from transmitting data and instead transmit reference signals for wideband channel estimation over one TTI. After that, each HeNB estimates its minimum resource demand and identifies the interfering HeNBs. The resource demand and interference information is then reported to the HMS for executing the proposed scheme in the next TTI. It is noteworthy that the channel measurements obtained from channel estimation done over one TTI in each period of $T_{\mathrm{RA}}$ may not be fully accurate to represent the long-term channel conditions as the channel could vary rapidly over time. To address this inaccuracy issue, the respective moving averages of the estimated interference and resource demands are calculated and reported to the HMS. In the implementation of our proposed scheme, the estimated interference and resource demands are averaged using a moving average window of size $W_{\mathrm{mv}}$. In other words, the estimated interference and resource demand of an HeNB obtained from the previous $W_{\mathrm{mv}}$ channel estimates in which each is done over one TTI in an interval of $T_{\mathrm{RA}}$ are stored, and the respective average values of the estimated interference and resource demands are calculated and reported to the HMS. After executing the proposed scheme, each HeNB will receive a subset of subchannels, which will be used for the next $T_{\mathrm{RA}}$. It is noteworthy that resource allocation among the data flows in the eNB as well as that in each HeNB depend on their scheduling policy. In this work, we assume that the proportional fair scheduling policy is implemented at both eNB and HeNBs to achieve a balance between fair resource allocation and high throughput among the MUEs served by the eNB and that among the FUEs served by each HeNB [27].

\section{COMPlEXity AND OVERHEAd AnAlysis}

This section analyzes the complexity of the proposed twophase resource allocation scheme and its required amount of overhead. Two prominent resource allocation schemes, namely the C-DFP [9] and DRA [14] schemes are used for comparison with the proposed scheme. These two schemes are used for comparison because their mechanisms are mainly used for resource allocation among femtocells, and resource allocation among data flows is performed at the HeNB based on the scheduling policy implemented; which is similar to the proposed scheme.

\section{A. Complexity}

In the C-DFP scheme, the central entity requires a maximum of $|\mathcal{H}|^{2}|\mathcal{K}|^{2}$ iterations to complete resource allocation among all the HeNBs. Thus, it has an asymptotic time complexity of $\mathcal{O}\left(|\mathcal{H}|^{2}|\mathcal{K}|^{2}\right)$.

In the DRA scheme, resources are allocated as PRB pairs. For worst-case complexity analysis, we assume that each HeNB can utilize all the PRB pairs. The DRA scheme is executed every one LTE/LTE-A frame, thus there are $10|\mathcal{K}|$ PRB pairs available for allocation because each frame contains 10 TTIs and $|\mathcal{K}|$ subchannels. The DRA scheme allows each HeNB $h$ to obtain a number of PRB pairs which is equal to the total number of PRB pairs available divided by its number of interfering HeNBs, $N_{h, \mathrm{I}}$. As such, the DRA scheme requires $\frac{(10|\mathcal{H}||\mathcal{K}|)}{N_{h, \mathrm{I}}}$ iterations for all the HeNBs. Therefore, the DRA scheme has an asymptotic time complexity of $\mathcal{O}(|\mathcal{H}||\mathcal{K}|)$.

In the proposed two-phase resource allocation scheme, steps 1-5 in Algorithm 2 require $\left|\mathcal{H}_{l}\right|$ iterations to complete for each femtocell group. For worst-case analysis, we assume that all subchannels are allocated to the femtocell group and each HeNB has a minimum resource demand that equates the total channel bandwidth available, i.e., $\mathcal{K}_{l}=\mathcal{K}$. Assume that the steps 1-5 are not performed and each HeNB has a minimum resource demand that equates the total channel bandwidth available, i.e., $\mathcal{K}_{\mathrm{r}, h}=\mathcal{K}$ and $D_{h}=|\mathcal{K}|$, the maximum number of iterations required for steps 6-30 in Algorithm 2 is calculated as $\frac{\left|\mathcal{H}_{l}\right||\mathcal{K}|^{2}+\left|\mathcal{H}_{l}\right||\mathcal{K}|}{2}$. Similarly, the maximum number of iterations required by Algorithm 3 is also $\frac{\left|\mathcal{H}_{l}\right||\mathcal{K}|^{2}+\left|\mathcal{H}_{l}\right||\mathcal{K}|}{2}$. As $\sum_{l \in \mathcal{L}}\left|\mathcal{H}_{l}\right|=|\mathcal{H}|$, the sum maximum number of iterations required by the proposed scheme is $\frac{|\mathcal{H}||\mathcal{K}|^{2}+|\mathcal{H}||\mathcal{K}|}{2}$. Thus, the proposed scheme has an asymptotic time complexity of $\mathcal{O}\left(|\mathcal{H}||\mathcal{K}|^{2}\right)$.

It is evident that the asymptotic time complexity of the proposed scheme is lower than that of the C-DFP scheme, but higher than that of the DRA scheme. Nevertheless, as will be demonstrated in Section VII, the proposed scheme can provide significant performance improvements over the DRA scheme.

\section{B. Overhead}

To analyze and compare the amount of signaling overhead required by all the schemes for information exchange, we assume an identical execution period for all the schemes. In the C-DFP scheme, the central controller collects the interference and resource demand information from the femtocells for resource allocation. Assuming that the resource demand does not exceed the number of available resources, it can then be represented by $\left\lceil\log _{2}|\mathcal{K}|\right\rceil$ bits. Thus, the signaling overhead required is $|\mathcal{H}|\left\lceil\log _{2}|\mathcal{K}|\right\rceil+I|\mathcal{H}|+I|\mathcal{H}|^{2}$ bits where $I$ is 
the number of bits required to represent the interference information from one HeNB. After resource allocation, the central entity sends the resource information to the HeNBs. Since the allocation of each resource can be represented by one bit, the signaling overhead required is $|\mathcal{H}||\mathcal{K}|$ bits. Hence, the total signaling overhead required by the C-DFP scheme is $I|\mathcal{H}|^{2}+I|\mathcal{H}|+|\mathcal{H}||K|+|\mathcal{H}|\left\lceil\log _{2}|\mathcal{K}|\right\rceil$ bits.

In the DRA scheme, the network controller collects the information regarding the numbers of MUEs and FUEs for spectrum partitioning. Thus, assuming that the number of bits required to represent the number of users is $U$, the signaling overhead required is $U(1+|\mathcal{H}|)$ bits. After spectrum partitioning, the central controller transmits the resource information to the eNB and HeNBs, which requires a signaling overhead of $|\mathcal{K}|(1+|\mathcal{H}|)$ bits. Hence, the total signaling overhead required by the DRA scheme is $U+|\mathcal{K}|+U|\mathcal{H}|+|\mathcal{H}||\mathcal{K}|$ bits.

In our proposed scheme, the eNB collects the resource demand information from each nearby interfering femtocell group through their HMSs for split spectrum allocation between the macrocell and the nearby interfering femtocell groups. After spectrum allocation, the eNB sends the resource information to the HMSs. Thus, the signaling overhead required is $\left|\mathcal{L}_{\mathrm{i}}\right|\left\lceil\log _{2}|\mathcal{K}|\right\rceil+\left|\mathcal{L}_{\mathrm{i}}\right||\mathcal{K}|$ bits where $\mathcal{L}_{\mathrm{i}}$ is the set of HMSs of the interfering femtocell groups. The signaling overhead required when each HMS collects the interference and resource demand information from the connected HeNBs is $I\left|\mathcal{H}_{l}\right|^{2}+\left|\mathcal{H}_{l}\right|\left\lceil\log _{2}|\mathcal{K}|\right\rceil$ bits. After resource allocation, each HMS sends the resource information to each of its connected HeNBs where the signaling overhead required is $\left|\mathcal{H}_{l}\right||\mathcal{K}|$ bits. Since $\sum_{l \in \mathcal{L}}\left|\mathcal{H}_{l}\right|=|\mathcal{H}|$, the total signaling overhead required by the proposed scheme for all the HMSs is $I \sum_{l \in \mathcal{L}}\left|\mathcal{H}_{l}\right|^{2}+|\mathcal{H}||\mathcal{K}|+|\mathcal{H}|\left\lceil\log _{2}|\mathcal{K}|\right\rceil+\left|\mathcal{L}_{\mathrm{i}}\right|\left\lceil\log _{2}|\mathcal{K}|\right\rceil+\left|\mathcal{L}_{\mathrm{i}}\right||\mathcal{K}|$ bits.

To compare the signaling overhead incurred by the three schemes, we assume that $I=1$ bit, $U=7$ bits for encoding up to $100 \mathrm{UEs}$ and $|\mathcal{K}|=25$. Also, for the proposed scheme, we assume that all the femtocell groups have equivalent numbers of HeNBs, i.e., $\left|\mathcal{H}_{l}\right| \approx\left|\mathcal{H}_{v}\right|$ for all $v \in \mathcal{L} \backslash\{l\}$. Fig. 3 illustrates the signaling overhead incurred by each of the schemes with respect to the total number of HeNBs in a cellular network, i.e., $|\mathcal{H}|$. The C-DFP scheme incurs the highest signaling overhead whereas the DRA scheme incurs the lowest signaling overhead. The proposed scheme with one HMS incurs signaling overhead which is almost as high as that of the CDFP scheme because the HMS serves all the HeNBs, which is basically equivalent to the central controller in the C-DFP scheme. However, the proposed scheme with more than one HMS requires significantly less signaling overhead because each HMS serves a smaller number of HeNBs. Besides, an increase in the number of interfering femtocell groups does not significantly increase the signaling overhead of the proposed scheme.

\section{ViI. Performance Evaluation}

The proposed two-phase resource allocation scheme is evaluated using the open-source LTE simulator [35], [36]. We compare the proposed scheme with two existing prominent

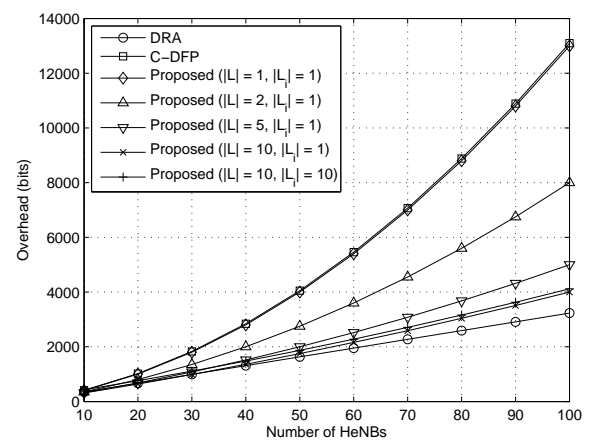

Fig. 3. Signaling overhead comparison between the proposed scheme and the existing schemes.

schemes, namely the C-DFP [8] and DRA [14] schemes. Also, we compare the performance of the proposed scheme, which provides the resource allocation solution to (7), with the exhaustive search, which returns a Pareto-optimal solution. We first introduce the performance metrics used, followed by the simulation results and discussion.

\section{A. Performance Metrics}

Several performance metrics are used to evaluate the three schemes, as follows:

1) Throughput: In downlink, the throughput achieved by a cellular network is defined as the number of bits successfully received by all the UEs over a time period. In our performance evaluation, throughput will be evaluated for GBR flows and non-GBR flows separately.

2) Packet Loss Rate: PLR is a widely use QoS metric for GBR flows, which indicates the percentage of packet loss during transmission in a cellular network.

3) Throughput Satisfaction Ratio: Throughput satisfaction ratio (TSR) is a QoS metric introduced in [20], which is given as $\operatorname{TSR}(h)=\frac{\sum_{k \in \mathcal{K}} \omega_{h k}}{D_{h}}$. If the minimum resource demand of $\mathrm{HeNB} h$ is fully satisfied, then $\operatorname{TSR}(h)=1$. We further extend this definition to accommodate over-satisfaction cases where HeNBs receive an amount of subchannels more than their minimum resource demand. In other words, if $\operatorname{TSR}(h)$ $>1$, this implies that HeNB $h$ is over-satisfied. The average TSR of a femtocell network can be calculated as $\frac{\sum_{h \in \mathcal{H}} \operatorname{TSR}(h)}{|\mathcal{H}|}$.

4) Jain's Fairness Index: To evaluate global fairness among femtocells, we employ Jain's fairness index [37], which is defined as $\frac{\left(\sum_{h \in \mathcal{H}} \operatorname{TSR}(h)\right)^{2}}{|\mathcal{H}| \sum_{h \in \mathcal{H}}(\operatorname{TSR}(h))^{2}}$. The values of Jain's fairness index range within $(0,1]$ where a large index value represents high fairness.

5) Resource Utilization Efficiency: To assess resource utilization of all femtocells, we define the resource utilization efficiency as $\frac{\sum_{h \in \mathcal{H}} \sum_{h \in \mathcal{K}} \omega_{h k}}{|\mathcal{H}||\mathcal{K}|}$.

\section{B. Performance Comparison between the Proposed Scheme and Existing Schemes}

For performance comparison between the proposed scheme and existing schemes, we simulate a single-macrocell LTE 
TABLE I

LTE Femtocell Network Simulation Setting

\begin{tabular}{c||c}
\hline \multicolumn{1}{c||}{ Parameter } & Setting \\
\hline \hline Frame Structure & Frequency division duplexing \\
Bandwidth & $5 \mathrm{MHz}(25$ subchannels $)$ \\
Simulation Duration & $30 \mathrm{~s}$ \\
Traffic Model & Video and best effort \\
Maximum Delay for GBR Flows & $0.1 \mathrm{~s}$ \\
HeNBs' Transmission Power & $20 \mathrm{dBm}$ (equally distributed \\
Scheduler & among subchannels) \\
Building Type & Proportional fair \\
Number of Buildings & $5 \times 5$ grid \\
Number of Apartments per Building & 2 \\
Apartment Size & 25 \\
Path Loss Model & $10 \times 10 \mathrm{~m}^{2}$ \\
Channel Fading Model & Urban indoor propagation \\
Shadowing & model (Winner) [36] $)$ \\
Femtocell Access Mode & Rayleigh \\
\multicolumn{2}{c}{ Lognormal } \\
\hline
\end{tabular}

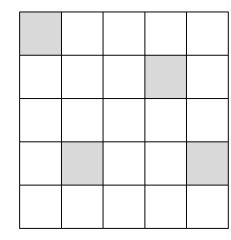

$r=0.2$

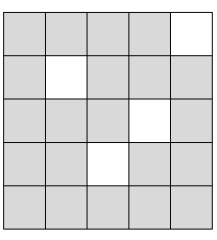

$r=0.8$

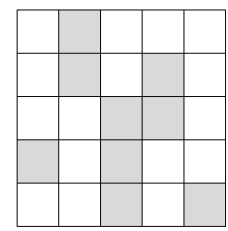

$r=0.4$
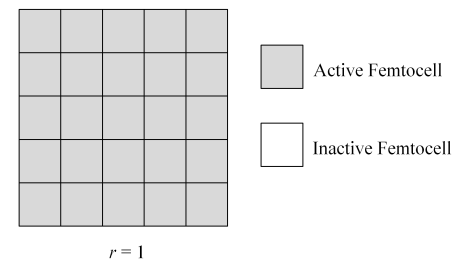

Fig. 4. Active femtocells in random locations with different $r$ in a building.

network with only two indoor buildings each forming a femtocell group, i.e., $|\mathcal{L}|=2$. One of the buildings is located near the eNB and the other is located far from the eNB, thus $\left|\mathcal{L}_{\mathrm{i}}\right|=1$. Each of the buildings is of two-dimensional and $5 \times 5$ apartment grid type [38]. Each apartment in the building has an area of $10 \times 10 \mathrm{~m}^{2}$ and accommodates a femtocell. Each HeNB in the building (femtocell group) is connected to an HMS. Within each femtocell, each FUE carries a video flow and a best-effort flow. The minimum bit rate of each video flow is set to $128 \mathrm{kbps}$. For the best-effort flow, an infinite buffer model is used. For the C-DFP scheme and the proposed scheme, $T_{\mathrm{RA}}$ is set to $1 \mathrm{~s}$, and $T h$ is set to 12 $\mathrm{dB}$ as in [28] for identifying the interfering HeNBs. For the proposed scheme, $T_{\text {alloc }}$ and $W_{\mathrm{mv}}$ are also set to $1 \mathrm{~s}$ and 10 , respectively. The focus of this paper is to demonstrate the effectiveness of the proposed scheme in allocating resources to HeNBs. Therefore for fair comparisons, all the schemes are assumed to use the conventional proportional fair packet scheduler for resource allocation among the FUEs within each femtocell. Other parameters used are shown in Table I. The simulation results obtained are averaged over five runs with different random number seeds at each run.

Within each building, we consider that a random number of femtocells are deployed or activated in random locations. The activity ratio, $r$ [38], which is defined as the probability of an HeNB is active in the building, is used as an indicator to represent the density of randomly located femtocells within the building. Fig. 4 illustrates the active femtocells in random locations with different $r$. In this study, we set $r=0.2$ and $r=0.6$ to represent low-density and high-density femtocell deployment scenarios, respectively. The resource allocation schemes are evaluated under Scenarios A and B. In Scenario A, each femtocell contains a fixed number of FUEs. We further evaluate this scenario with the macrocell having one and ten MUEs. We denote the former and latter as Scenarios A1 and A2, respectively. In Scenario B, each femtocell has a random number of up to five FUEs and the macrocell has a random number of up to ten MUEs.

Fig. 5(a) shows the throughput performance in Scenario A1 with $r=0.2$. In this scenario, the proposed scheme and the C-DFP scheme achieve comparable video throughput performance. It can be further observed in Fig. 5(c) that the proposed scheme achieves a lower video PLR compared to the C-DFP scheme in the same scenario. Also, the proposed scheme achieves the highest best effort throughput performance in the scenario, as shown in Fig. 5(a). This is due to low co-tier interference in the scenario which allows the proposed scheme to further increase and maximize the number of resources allocated to each HeNB, thereby maximizing resource utilization efficiency. The DRA scheme initially attains comparable throughput and PLR performance with other two schemes, as shown in Figs. 5(a) and 5(c), respectively. However, its performance starts to deteriorate with increased numbers of FUEs per femtocell because it could not provide sufficient resources to large numbers of FUEs.

Fig. 5(b) shows the throughput performance in Scenario A1 with $r=0.6$. In this scenario, the throughput performance of the proposed scheme is equivalent to that of the C-DFP scheme. This is due to high co-tier interference in the scenario which does not allow the proposed scheme to further increase the amount of resources allocated to each HeNB. As a result, it can only provide the amount of resources that is sufficient to meet the resource demand of each HeNB. This is equivalent to the resource allocation mechanism of the C-DFP scheme. Again, the throughput performance of the DRA scheme declines as the number of FUEs per femtocell increases. The aforementioned performance trends are in line with the video PLR performance in Fig. 5(c).

The throughput and PLR performance in Scenario A2 are illustrated in Figs. 5(d), 5(e) and 5(f). Figs. 5(d) and 5(e) show a similar throughput performance trends as those in Scenario A1. It can be observed from Fig. 5(f) that the proposed scheme is slightly inferior to the C-DFP scheme in terms of video PLR when $r=0.6$. This is because the macrocell has more MUEs in Scenario A2, which requires more resources. As a result, the proposed scheme allocates less channel bandwidth to the femtocell group which is near the eNB.

Fig. 6(a) shows the average TSR achieved by the three schemes in Scenario A1. When $r=0.2$, the proposed scheme generally outperforms the other two schemes because it increases and maximizes the number of resources allocated 


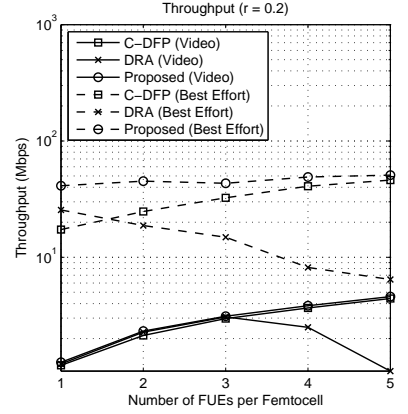

(a)

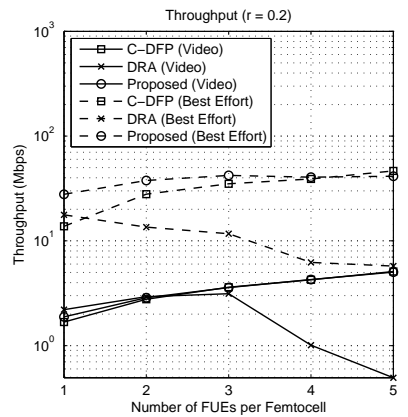

(d)

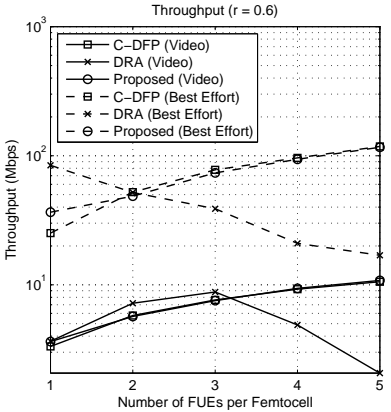

(b)

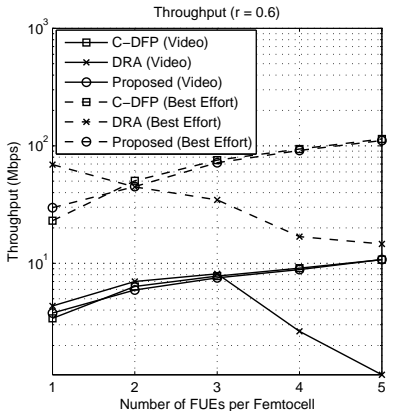

(e)

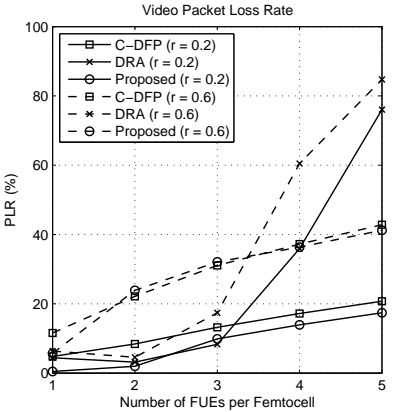

(c)

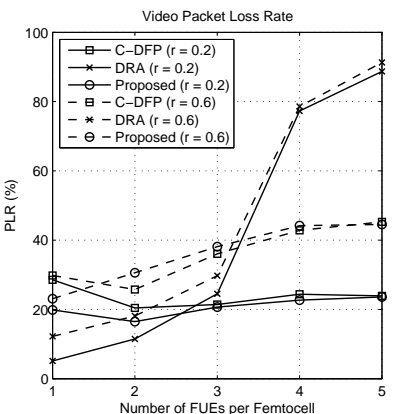

(f)

Fig. 5. Throughput performance in Scenario A1 with (a) $r=0.2$ and (b) $r=0.6$. (c) Video PLR performance in Scenario A1. Throughput performance in Scenario A2 with (d) $r=0.2$ and (e) $r=0.6$. (f) Video PLR performance in Scenario A2.

to the HeNBs. The DRA scheme is better than the proposed scheme only when the number of FUEs per femtocell is one. On the other hand, when $r=0.6$, the DRA scheme has a higher TSR than the other two schemes initially. However, its performance is inferior with larger numbers of FUEs per femtocell. The TSR achieved by the proposed scheme is equivalent to that of the C-DFP scheme when $r=0.6$ due to high co-tier interference in which no additional interferencefree resources can be allocated to the HeNBs. The C-DFP scheme constantly achieves $\operatorname{TSR}(\mathrm{h}) \approx 1$ for all $h$ because it only allocates HeNBs the amount of resources that is sufficient to meet their minimum resource demands.

Fig. 6(b) shows the global fairness performance of the network in Scenario A1. The C-DFP scheme maintains a maximum global fairness because it achieves $\operatorname{TSR}(\mathrm{h}) \approx 1$ for all $h$. When $r=0.2$, The DRA scheme initially achieves a higher level of global fairness than the proposed scheme but its global fairness declines with the number of FUEs per femtocell because some HeNBs receive insufficient or excessive resources. The proposed scheme maintains a relatively high level of global fairness exceeding 0.8 over all the numbers of FUEs per femtocell due to constraints (7a) and (7c) formulated in Problem 1. When $r=0.6$, the proposed scheme is almost comparable with the C-DFP scheme because the former also achieves $\operatorname{TSR}(\mathrm{h}) \approx 1$ for all $h$. In the same scenario, the DRA scheme is the worst performer.

Fig. 6(c) shows that the proposed scheme achieves the highest resource utilization efficiency among all the schemes in Scenario A1. This is because it maximizes the number of resources allocated to each HeNB. As a result, the throughput and PLR performance of the proposed scheme improves over the other two schemes when $r=0.2$. Although the proposed scheme attains a slightly higher resource utilization efficiency than the C-DFP scheme when $r=0.6$, the proposed scheme does not outperform the C-DFP scheme. This is because the higher resource utilization efficiency attained in highdensity femtocell deployment scenarios result in higher cotier interference. Also, the high resource utilization efficiency attained by the proposed scheme is mainly attributed to the high resource reuse in the distant femtocell group which shares the entire spectrum. Therefore, no performance improvements can be attained in the femtocell group which is near the eNB.

Figs. 6(d), 6(e) and 6(f) demonstrate similar performance trends in terms of average TSR, global fairness and resource utilization efficiency under Scenario A1 as those in Scenario A2. A main difference is observed where the performance of the DRA scheme in Scenario A2 becomes more inferior to that in Scenario A1. This is because more resources are allocated to the macrocell, hence leaving less resources for the femtocells.

The simulation results for Scenario B are tabulated in Table II. When $r=0.2$, the proposed scheme outperforms the other two schemes in terms of video and best effort throughput as well as video PLR. The proposed scheme also achieves the highest average TSR and maintains a relatively high level of global fairness at 0.8103 . It is noteworthy that the global fairness performance of the DRA scheme is much inferior to the other two schemes due to the fact that the TSR of the HeNBs is very much different among each other. In other words, some HeNBs receive excessive resources while others 


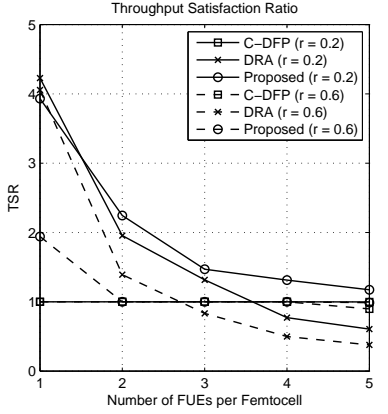

(a)

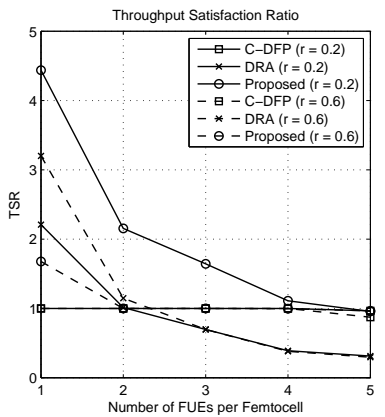

(d)

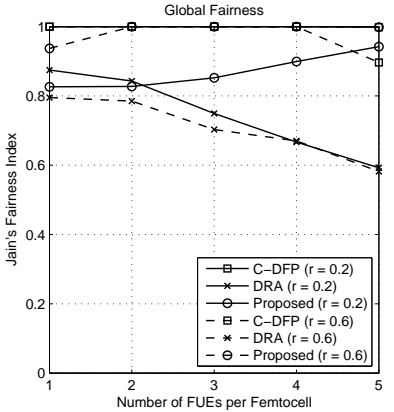

(b)

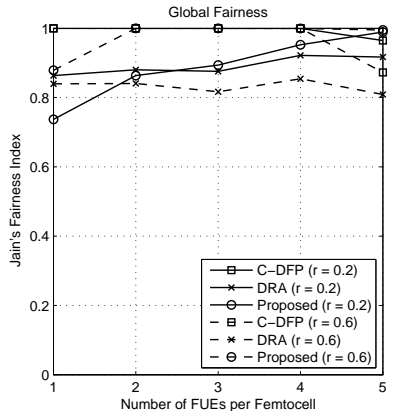

(e)

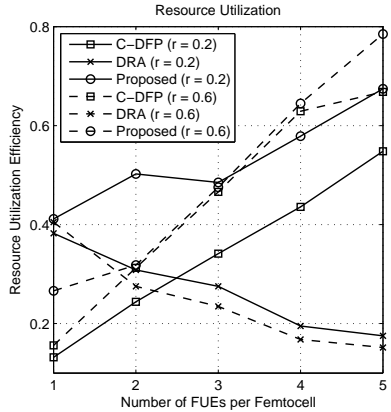

(c)

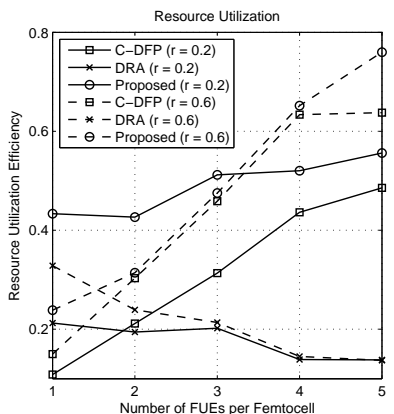

(f)

Fig. 6. (a) Average TSR, (b) global fairness and (c) resource utilization efficiency performance in Scenario A1. (d) Average TSR, (e) global fairness and (f) resource utilization efficiency performance in Scenario A2.

TABLE II

RESULTS FOR SCENARIO B

\begin{tabular}{c|ccc||ccc}
\hline \hline \multirow{2}{*}{ Metric } & \multicolumn{3}{|c||}{$r=0.2$} & \multicolumn{2}{c}{$r=0.6$} \\
\cline { 2 - 6 } & C-DFP & DRA & Proposed & C-DFP & DRA & Proposed \\
\hline \hline Video Throughput & $3.424 \mathrm{Mbps}$ & $2.1828 \mathrm{Mbps}$ & $3.499 \mathrm{Mbps}$ & $7.654 \mathrm{Mbps}$ & $5.0147 \mathrm{Mbps}$ & $7.3429 \mathrm{Mbps}$ \\
Best Effort Throughput & $35.205 \mathrm{Mbps}$ & $12.985 \mathrm{Mbps}$ & $42.425 \mathrm{Mbps}$ & $82.909 \mathrm{Mbps}$ & $40.626 \mathrm{Mbps}$ & $81.256 \mathrm{Mbps}$ \\
Video PLR & $12.9558 \%$ & $39.3655 \%$ & $10.8374 \%$ & $35.0244 \%$ & $53.7469 \%$ & $37.4014 \%$ \\
Average TSR & 1 & 1.0425 & 1.7782 & 0.9929 & 1.0338 & 0.9914 \\
Global Fairness & 1 & $0.2332 \times 10^{-4}$ & 0.8103 & 0.9929 & $0.1773 \times 10^{-4}$ & 0.9988 \\
Resource Utilization Efficiency & 0.3598 & 0.2129 & 0.4768 & 0.4641 & 0.2314 & 0.4717 \\
\hline \hline
\end{tabular}

have insufficient resources. The proposed scheme achieves the highest resource utilization efficiency because it maximizes the number of resources allocated to each HeNB, resulting in the superior throughput and PLR performance. When $r=0.6$, the proposed scheme is slightly inferior to the C-DFP scheme in terms of throughput and video PLR while the DRA scheme remains the most inferior performer. Both the proposed scheme and the C-DFP scheme attain comparable TSR and global fairness. It is observed that the resource utilization efficiency achieved by the proposed scheme is higher than that of the C-DFP scheme.

We can conclude that performance gains can be obtained by the proposed scheme in low-density femtocell deployment scenarios thanks to its ability of maximizing the number of resources allocated to each HeNB. A relatively high level of global fairness and resource utilization efficiency are also achieved by the proposed scheme. In high-density femtocell deployment scenarios, the number of resources allocated to each HeNB cannot be increased further due to high co-tier interference. Therefore, the proposed scheme can only allocate sufficient resources to each $\mathrm{HeNB}$ to meet its minimum resource demand. This justifies the equivalent performance of the proposed scheme and the C-DFP scheme. Compared to the C-DFP scheme, although the proposed scheme incurs a small throughput and PLR performance loss in high-density femtocell deployment scenarios, this performance loss is compensated by a huge reduction in computational complexity and signaling overhead as shown in Section VI. It is worth noting that the DRA scheme is efficient only when the number of FUEs per femtocell is small and the achieved global fairness level is very low when the number of FUEs per femtocell is random.

\section{Performance Comparison between the Pareto-Optimal So- lution and the Proposed Solution}

For performance comparison between the Pareto-optimal solution obtained by exhaustive search and the solution obtained by the proposed scheme, we consider a single-macrocell LTE 
network with one indoor building. Due to the heavy computational burden required by the exhaustive search method for the optimal solution to (7), we consider the smaller indoor building of $2 \times 2$ apartment grid type comprising four apartments with each housing one femtocell. Each HeNB in the building is connected to an HMS. To reduce computational burden, we set the channel bandwidth as $2 \mathrm{MHz}$ which contains ten subchannels. The rest of the parameters are set following Table I. The simulation results obtained are averaged over five runs where each run realizes different channel conditions. In the comparison between the Pareto-optimal solution and the proposed solution, we consider a scenario with an increasing number of up to three randomly active femtocells ${ }^{3}$ within the building and each active femtocell contains two FUEs.

It is noteworthy that both solutions will provide the same TSR, global fairness and resource utilization. Therefore, we only examine their throughput and PLR performance. In Fig. 7(a), it is demonstrated that the video throughput performance achieved by the proposed solution closely approximates that achieved by the Pareto-optimal solution obtained by exhaustive search for different numbers of femtocells, though the former being slightly inferior. Similarly, Fig. 7(b) shows that the best-effort throughput performance achieved by the proposed solution is nearly identical to that achieved by the Paretooptimal solution. The video PLR performance in Fig. 7(c) is in line with the video throughput performance in Fig. 7(a), whereby the Pareto-optimal solution only attains an PLR of approximately $1 \%$ less than that of the proposed solution for different numbers of femtocells. These observations indicate that the performance gap between the Pareto-optimal solution and the proposed solution is very small. This indicates that the proposed scheme can achieve performance comparable to the Pareto-optimal solution albeit with substantial computational time reduction.

\section{CONCLUSION}

This paper proposed a new multi-objective resource allocation scheme under a hybrid spectrum allocation approach for LTE/LTE-A femtocell networks. The hybrid spectrum allocation approach splits the spectrum among the eNB and its nearby interfering HeNBs while the distant HeNBs share the entire spectrum. A multi-objective optimization problem has been formulated for resource allocation between femtocells to jointly minimize co-tier interference and maximize resource utilization efficiency while guaranteeing a high level of global fairness. The problem is further decomposed into two subproblem using a lexicographic optimization approach and the solution obtained from solving these subproblems sequentially has been proven to be Pareto optimal for the original problem. A two-phase greedy algorithm has been devised to solve the two subproblems. The proposed scheme has been shown to have a lower asymptotic complexity and requires less signaling overhead than the C-DFP scheme although the DRA scheme has an even lower complexity and needs less signaling overhead. Simulation results have shown that the proposed scheme

\footnotetext{
${ }^{3}$ The amount of the computational time required by exhaustive search in the scenario with more than three femtocells is prohibitively large. Thus, we only simulate the scenario with up to three randomly active femtocells.
}

achieves substantial throughput and PLR performance gains over the existing schemes in low-density femtocell deployment scenarios; while performing almost equivalently to the $\mathrm{C}$ DFP scheme in high-density femtocell deployment scenarios albeit with a significantly lower complexity and less signaling overhead. The proposed scheme also maintains a relatively high level of global fairness and resource utilization efficiency; therefore it is promising for use in LTE femtocell networks. In addition, the performance of the resource allocation solution obtained by the proposed scheme is nearly identical to that of the Pareto-optimal solution obtained by exhaustive search. Although the proposed scheme is designed based on the LTE system architecture, it is also applicable to LTE-A systems. The PLR performance can be further improved by means of scheduling and admission control, which will be left for future work.

\section{ACKNOWLEDGMENT}

The authors would like to thank Mr. Vishnu Monn Baskaran and Dr. Chee Keong Tan from Multimedia University, Malaysia, and Dr. Yu-Fan Chen and Prof. Li-Chun Wang from National Chiao Tung University, Taiwan, for their assistance in providing computing facilities to help complete this work. The authors would also like to thank the editor and the anonymous reviewers for their constructive comments and suggestions to improve the quality of this manuscript.

\section{REFERENCES}

[1] Y. L. Lee, T. C. Chuah, J. Loo, and A. Vinel, "Recent Advances in Radio Resource Management for Heterogeneous LTE/LTE-A Networks," IEEE Commun. Surveys Tuts., vol. 16, no. 4, pp. 2142 - 2180, June 2014.

[2] V. Chandrasekhar, J. G. Andrews, and A. Gatherer,"Femtocell Networks: A Survey" IEEE Commun. Mag., vol. 46, no. 9, pp. 59 - 67, Sep. 2008.

[3] T.-H. Kim, and T.-J. Lee, "Throughput Enhancement of Macro and Femto Networks by Frequency Reuse and Pilot Sensing" in Proc. IEEE IPCCC, Austin, Texas, US, Dec. 2008, pp. 390 - 394.

[4] N. Saquib, E. Hossain, L. B. Le, and D. I. Kim, "Interference Management in OFDMA Femtocell Networks: Issues and Approaches," IEEE Wireless Commun., vol. 19, no. 3, pp. 86 - 95, June 2012.

[5] D. Lopez-Perez, I. Guvenc, G. de la Roche, M. Kountouris, T. Q. S. Quek, and J. Zhang, "Enhanced Intercell Interference Coordination Challenges in Heterogeneous Networks," IEEE Wireless Commun., vol. 18, no. 3, pp. 22 - 30, June 2011.

[6] D. Lopez-Perez, I. Guvenc, and X. Chu, "Mobility Management Challenges in 3GPP Heterogeneous Networks," IEEE Commun. Mag., vol. 50, no. 12 , pp. $70-78$, Dec. 2012.

[7] M. C. Erturk, I. Guvenc, S. Mukherjee and H. Arslan, "Fair and QoSOriented Resource Management in Heterogeneous Networks," EURASIP J. Wireless Commun. Netw., vol. 2013, no. 121, pp. 1 - 14, May 2013.

[8] Z. Zheng, A. A. Dowhuszko and J. Hamalainen, "Interference Management for LTE-Advanced Het-Nets: Stochastic Scheduling Approach in Frequency Domain," Trans. Emerg. Telecommun. Technol., vol. 24, no. 1, pp. 4 17, Jan. 2013.

[9] D. Lopez-Perez, A. Valcarce, G. de la Roche and J. Zhang, "OFDMA Femtocells: A Roadmap on Interference Avoidance," IEEE Commun. Mag., vol. 47, no. 9, pp. 41 - 48, Sep. 2009.

[10] Z. Lu, T. Bansal and P. Sinha, "Achieving User-Level Fairness in OpenAccess Femtocell-Based Architecture," IEEE Trans. Mobile Comput., vol. 12, no. 10, pp. 1943 - 1954, Oct. 2013.

[11] M. E. Sahin, I. Guvenc, M.-R. Jeong, and H. Arslan, "Handling CCI and ICI in OFDMA Femtocell Networks Through Frequency Scheduling," IEEE Trans. Consum. Electron., vol. 55, no. 4, pp. 1936 - 1944, Nov. 2009.

[12] S.-Y. Lien, Y.-Y. Lin, and K.-C. Chen, "Cognitive and Game-Theoretical Radio Resource Management for Autonomous Femtocells with QoS Guarantees," IEEE Trans. Wireless Commun., vol. 10, no. 7, pp. 2196 - 2206, July 2011. 


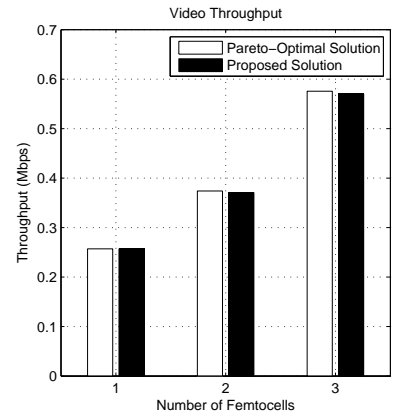

(a)

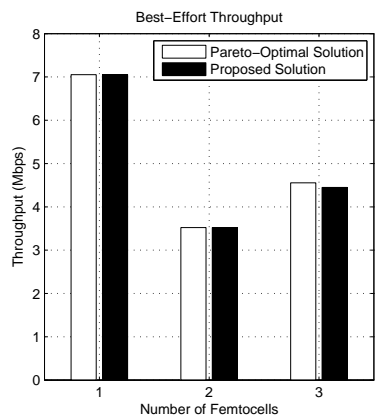

(b)

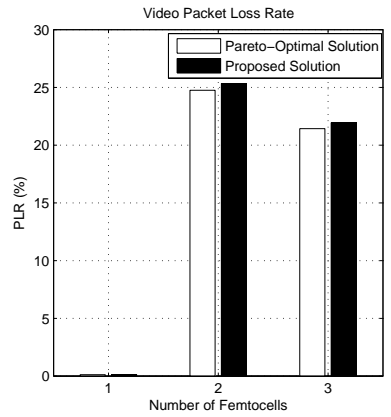

(c)

Fig. 7. (a) Video throughput, (b) best-effort throughput and (c) video PLR performance of the Pareto-optimal solution and the proposed solution.

[13] O. N. Ghareshiran, A. Attar and V. Krishnamurthy, "Collaborative SubChannel Allocation in Cognitive LTE Femto-Cells: A Cooperative Game Theoretic Approach," IEEE Trans. Commun., vol. 61, no. 1, pp. 325 334, Jan. 2013

[14] K. Sundaresan and S. Rangarajan, "Efficient Resource Management in OFDMA Femtocells," in Proc. ACM MobiHoc, New Orleans, Louisiana, USA, May 2009, pp. $33-42$.

[15] K. Lee, O. Jo, and D.-H. Cho, "Cooperative Resource Allocation for Guaranteeing Intercell Fairness in Femtocell Networks," IEEE Commun. Lett., vol. 15, no. 2, pp. 214 - 216, Feb. 2011.

[16] D. Lopez-Perez, X. Chu, A. V. Vasilakos, H. Claussen, "Power Minimization Based Resource Allocation for Interference Mitigation in OFDMA Femtocell Networks," IEEE J. Sel. Areas Commun., vol. 32, no. 12, pp. 333 - 344, May 2013.

[17] R. K. Saha, "Modified Proportional Fair Scheduling for Resource Reuse and Interference Coordination in Two-Tier LTE-Advanced Systems," International J. Digital Inf. Wireless Commun., vol. 3, no. 2, pp. 9 28, 2013.

[18] Y.-S. Liang, W.-H. Chung, G.-K. Ni, I.-Y. Chen, H. Zhang and S.-Y. Kuo, "Resource Allocation with Interference Avoidance in OFDMA Femtocell Networks," IEEE Trans. Veh. Technol., vol. 61, no. 5, pp. 2243 - 2255 , June 2012.

[19] B.-G. Kim, J.-A. Kwon and J.-W. Lee, "Subchannel Allocation for the OFDMA-Based Femtocell System," Comput. Netw., vol. 57, no. 17, pp. 3617 - 3629, Dec. 2013.

[20] A. Hatoum, R. Langar, N. Aitsaadi, R. Boutaba and G. Pujolle, "Clusterbased Resource Management in OFDMA Femtocell Networks with QoS Guarantees," IEEE Trans. Veh. Technol., vol. 63, no. 5, pp. 2378 - 2391, Nov. 2013.

[21] V. Chandrasekhar and J. Andrews, "Spectrum Allocation in Tiered Cellular Networks," IEEE Trans. Commun., vol. 57, no. 10, pp. 3059 - 3068, Oct. 2009.

[22] 3GPP, "Evolved Universal Terestrial Radio Access (E-UTRA) and Evolved Universal Terestrial Radio Access Network (EUTRAN); Overall description: Stage 2," 3rd Generation Partnership Project (3GPP), TS 36.300, Dec. 2013. [Online]. Available: http://www.3gpp.org/DynaReport/36.300.htm

[23] 3GPP, "Technical Specification Group Services and System Aspects; Telecommunication Management; Home enhanced Node B (HeNB) Operations, Administration, Maintenance and Provisioning (OAM\&P); Procedure flows for Type 1 interface HeNB to HeNB Management System (HeMS)," 3rd Generation Partnership Project (3GPP), TS 32.593, June 2014. [Online]. Available: http://www.3gpp.org/DynaReport/32593.htm

[24] D. T. Ngo and T. Le-Ngoc, Architectures of Small-Cell Networks and Interference Management. Springer, 2014.

[25] Y. Bai and L. Chen, "Hybrid Spectrum Arrangement and Interference Mitigation for Coexistence between LTE Macrocellular and Femtoell Networks," EURASIP J. Wireless Commun. Netw., vol. 2013, no. 56, pp. 1 - 15, Mar. 2013.

[26] 3GPP, “Technical Specification Group Radio Access Network; Evolved Universal Terrestrial Radio Access (E-UTRA); Physical Channels and Modulation," 3rd Generation Partnership Project (3GPP), TS 36.211, Sep. 2012. [Online]. Available: http://www.3gpp.org/DynaReport/36.211.htm

[27] F. Capozzi, G. Piro, L. A. Grieco, G. Boggia, and P. Camarda, "Downlink Packet Scheduling in LTE Cellular Networks: Key Design Issues and a Survey," IEEE Commun. Surveys Tuts., vol. 15, no. 2, pp. 678 - 700, May 2013.

[28] D. Lopez-Perez, X. Chu, and J. Zhang, "Dynamic Downlink Frequency and Power Allocation in OFDMA Cellular Networks," IEEE Trans. Commun., vol. 60, no. 10, pp. 2904 - 2914, Oct. 2012.

[29] I. Guvenc, M.-R. Jeong, F. Watanabe, and H. Inamura, "A Hybrid Frequency Assignment for Femtocells and Coverage Area Analysis for Co-Channel Operation," IEEE Commun. Lett., vol. 12, no. 12, pp. 880 882, Dec. 2008.

[30] V. Pareto, Manuale di Economica Politica, Societa Editrice Libraria. 1906; translated into English by A.S. Schwier as Manual of Political Economy, edited by A.S. Schwier and A.N. Page. A.M. Kelley. 1971.

[31] X.-S. Yang, Introduction to Mathematical Optimization - From Linear Programming to Metaheuristics. Cambridge International Science Publishing. 2008 .

[32] M. Ehrgott, Multicriteria Optimization. Springer. 2005.

[33] N. Sriniyas, and K. Deb, "Multiobjective Optimization using Nondominated Sorting in Genetic Algorithms," Evol. Comput., vol. 2, no. 3, pp. 221 - 248, Sep. 1994.

[34] K. Deb, A. Pratap, S. Agarwal, and T. Meyarivan, "A Fast and Elitist Multiobjective Genetic Algorithm: NSGA-II," IEEE Trans. Evol. Comput., vol. 6, no. 2, pp. 182 - 197, Apr. 2002.

[35] G. Piro, L. A. Grieco, G. Boggia, F. Capozzi, and P. Camarda, "Simulating LTE Cellular Systems: An Open-Source Framework," IEEE Trans. Veh. Technol., vol. 60, no. 2, pp. 498 - 513, Feb. 2011.

[36] F. Capozzi, G. Piro, L. A. Grieco, G. Boggia and P. Camarda, "On Accurate Simulations of LTE Femtocells using an Open Source Simulator," EURASIP J. Wireless Commun. Netw., vol. 2012, no. 328, pp. 1 - 13, Oct. 2012.

[37] R. Jain, The Art of Computer Systems Performance Analysis. John Wiley \& Sons, 1991.

[38] 3GPP, "Simulation Assumptions and Parameters for FDD HeNB RF Requirements", 3rd Generation Partnership Project (3GPP) Project Document R4-092042, July 2009. 